\title{
ERRATUM
}

Open Access

\section{Erratum to: Stochastic modelling of infectious diseases for heterogeneous populations}

\author{
Rui-Xing Ming ${ }^{1}$, Jiming Liu², William K. W. Cheung ${ }^{2}$ and Xiang Wan ${ }^{3 *}$
}

\section{Erratum}

After the publication of this work [1], we noticed an error the author's name Ji-Ming Liu should be spelt as Jiming Liu.

The original article was corrected.

The publisher apologises for this error.

\author{
Author details \\ ${ }^{1}$ School of Statistics \& Mathematics, Zhejiang Gongshang University, \\ Hangzhou, China. ${ }^{2}$ Department of Computer Science, Hong Kong Baptist \\ University, Kowloon Tong, Hong Kong. ${ }^{3}$ Department of Computer Science \& \\ Institute of Theoretical and Computational Study, Hong Kong Baptist University, \\ Kowloon Tong, Hong Kong.
}

Received: 22 February 2017 Accepted: 22 February 2017

Published online: 28 February 2017

\section{Reference}

1. Ming R-X, Liu J, Cheung WKW, Wan X. Stochastic modelling of infectious diseases for heterogeneous populations. Infect Dis Poverty. 2016;5:107.

\footnotetext{
* Correspondence: xwan@comp.hkbu.edu.hk

${ }^{3}$ Department of Computer Science \& Institute of Theoretical and Computational Study, Hong Kong Baptist University, Kowloon Tong, Hong Kong
} 